\title{
Epidemiology, antibiotic consumption and molecular characterisation of Staphylococcus aureus infections - data from the Polish Neonatology Surveillance Network, 2009-2012
}

Dorota Romaniszyn', Anna Różańska1*, Jadwiga Wójkowska-Mach', Agnieszka Chmielarczyk', Monika Pobiega', Paweł Adamski ${ }^{2}$, Ewa Helwich³, Ryszard Lauterbach", Maria Borszewska-Kornacka5, Ewa Gulczyńska ${ }^{6}$, Agnieszka Kordek ${ }^{7}$ and Małgorzata Bulanda ${ }^{1}$

\begin{abstract}
Background: Our aim was to determine and characterize S. aureus (SA) isolated from infections in newborns for antibiotic resistance, virulence factors, genotypes, epidemiology and antibiotic consumption.

Methods: Prospective surveillance of infections was conducted. Data about antibiotic treatment were analyzed. Antimicrobial susceptibility was assessed. PCR amplification was used to detect resistance and virulence genes. Typing methods such as PFGE, spa-typing and SCCmec were used.

Results: SA was found to be associated with $6.5 \%$ of infections. Methicillin-Resistant Staphylococcus aureus accounted for $32.8 \%$ of SA-infections. An incidence of MRSA-infections was 1.1/1000 newborns. MRSA-infections were diagnosed significantly earlier than MSSA-infections in these newborns (14th day vs. 23rd day $(p=0.0194)$ ). MRSA-infections increased the risk of newborn's death. Antibiotic consumption in both group was similar, but a high level of glycopeptides-usage for MSSA infections was observed.

In the MRSA group, more strains were resistant to erythromycin, clindamycin, gentamicin and amikacin than in the MSSA group. Hla gene was present in $93.9 \%$ of strains, and seg and sei in $65.3 \%$ of strains, respectively. One dominant clone was found among the 14 MRSA isolates. Fifteen strains belonging to SCCmec type IV were spa-t015 and one strain belonging to SCCmec type $V$ was spa-t011.
\end{abstract}

Conclusions: Results obtained in the study point at specific epidemiological situation in Polish NICU (more detailed studies are recommended).

High usage of glycopeptides in the MSSA infections treatment indicates the necessity of antimicrobial stewardship improvement and introducing molecular screening for early identification of infections.

\section{Background}

Systemic infections caused by Staphylococcus aureus (SA) are still common among newborns in neonatal intensive care units (NICUs) [1]. The danger of $S$. aureus is attributed to its resistance to many antibiotics and high virulence [2]. To our knowledge, there have been no studies on the epidemiology of SA in patients of

\footnotetext{
* Correspondence: rozanska@ifb.pl

${ }^{1}$ Chair of Microbiology, Jagiellonian University Medical College, 18 Czysta Street, Kracow 31-121, Poland

Full list of author information is available at the end of the article
}

NICUs in Poland. In fact, there have been no systemic surveillance of such infections in this vulnerable population.

The primary aim of this study was to determine and characterize $S$. aureus isolates from newborns according to resistance, virulence factors, genotypes and epidemiology. Secondly, we assessed the level of antibiotic usage and possible risk factors for SA infection (separately for Methicillin-sensitive SA (MSSA) and Methicillin-resistant SA (MRSA)). These factors included: clinical (use of devices: central/peripheral venous catheter - CVC/ $\mathrm{PVC}$, mechanical ventilation - MV, continuous positive 
airway pressure - CPAP, or total parenteral nutrition TPN, length of stay in hospital until infection, and breast/ trophic feeding) and demographic data.

\section{Methods}

Utilization of data collected in the Polish Neonatology Surveillance Network (PNSN) for scientific purposes was approved by the Bioethics Committee of Jagiellonian University Medical College - no. KBET/227/B/2012. All data entered into the electronic database and analyzed during this study were previously anonymised and deidentified. The PNSN is a prospective national surveillance system for the most relevant infections in VLBW infants (with birth weight $<1500 \mathrm{~g}$ ) in Poland. Details of the following variables were collected for all VLBW newborns: birth weight and gestational age, gender, multiple pregnacy, type of delivery and information of the situation in time of delivery, for example chorioamnionitis, general status of newborns by Apgar score: at 1 and 5 minutes and Critical Risk Index for Babies, CRIB) and others. Participation in PNSN is voluntary and confidential, the surveillance covered over $20 \%$ of VLBW infants born in the regions where the NICUs were located.

\section{Study population}

Prospective surveillance of infections was conducted between 2009-2012 at five tertiary academic NICUs that lead the perinatal care, in hospitals (designed by letters $\mathrm{A}, \mathrm{B}, \mathrm{C}, \mathrm{E}, \mathrm{F}$ ) that participated in the PNSN, and included 1768 newborns. The labor of 135 newborns was complicated by chorioamnionitis, while 429 infants were delivered after premature rupture of membranes (PROM). All episodes of infection were subjected to recording, regardless of the time of occurrence of the first symptoms. Case patients were defined according to Gastmeier et al. [3] with modification, as very low birth weight (VLBW) neonates who demonstrated clinical signs and/or symptoms of bloodstream infection (BSI) or pneumonia.

Early-onset infection was defined as infection diagnosed within 3 days after delivery. Device-associated infection was defined as infection diagnosed in newborns who had a device (central/peripheral venous catheter or mechanical ventilation or continuous positive airway pressure) placed within the 48-hour period before infection onset. Device utilization ratio (calculated by dividing the number of days with devices by the total number of patient/days, pds) was: 0.41 for CVC, 0.21 for PVC, 0.38 for MV, 0.28 for CPAP and 0.47 for TPN.

SA-infection was defined as culture-proven infection with isolation of SA. MSSA (or MRSA) infection was defined as culture-proven infection with isolation of MSSA (or MRSA).

\section{Bacterial isolates}

Collecting and identifying bacterial strains were performed in the local microbiology laboratories. Various diagnostic specimens were collected for culture and assessment of the microbial aetiology of infections. Altogether, 58 SA strains were isolated - and were considered to be the aetiological factor of infection. In this group, 49 strains were used for further analysis: most were from cases of pneumonia (20) and from BSI (19); nine were not stored. Strains in local laboratory were stored in $-20^{\circ} \mathrm{C}$, in laboratory of Chair of Microbiology were stored in $-70^{\circ} \mathrm{C}$. Isolates were identified by the automated identification system (VITEK 2, bioMerieux, Poland).

\section{Susceptibility testing}

All strains were tested using disk diffusion antimicrobial susceptibility methods according to current guidelines of the European Committee on Antimicrobial Susceptibility Testing (Clinical breakpoints tables v. 3.1; http://www. eucast.org v.3.1). E-tests for vancomycin and teicoplanin (bioMérieux, Paris, France) were also performed for all the isolates.

The MRSA resistance phenotype was detected using a cefoxitin disc $(30 \mu \mathrm{g})$ according to the EUCAST guidelines. The macrolide-lincosamide (MLS) resistance phenotype of the isolates was determined according to a previously published protocol [4].

\section{DNA isolation}

DNA was extracted from isolates using the Genomic Mini kit (A\&A Biotechnology, Gdynia, Poland) according to the manufacturer's instructions.

\section{Polymerase chain reaction (PCR) screening for resistance genes}

PCR amplification was used to detect the mecA gene using previously published primers [5]. As controls, S. aureus ATCC 33591 (mecA+) and S. aureus ATCC 25923 (mecA-) were employed. Erythromycin resistance genes (ermA, ermB, erm $\mathrm{C}$, and $m s r)$ were detected by multiplex PCR, and amplification of a $456 \mathrm{bp}$ fragment of the mupA gene (mupirocin resistance gene) was performed by single PCR [6,7].

\section{Antibiotic treatment}

Data about antibiotic treatment were entered into the database by the ward personnel based on the physicians' orders. Precise information about the type of drug, daily dose, and length of treatment for each antibiotic was collected and used for the calculation of two indicators: duration of treatment and defined daily dose in reference to each case of infection.

The aggregate sum of the number of days during which at least one dose of antibiotic was received for 
each antibiotic used (days of treatment, DOTs) was expressed in days and the defined daily dose (DDD), according to the ATC/DDD system of the World Health Organization (Anatomical Therapeutic Chemical, group "J01"). Only antibiotics for systemic use were taken into account.

Antibiotic usage for treatment (until cure) was assessed for 56 cases; two cases were excluded from analysis due to death of the infant within the first seven days of illness (unsuccessful treatment) and lack of data on treatment of one newborn.

\section{Virulence factor screening}

$S$. aureus isolates were checked for the presence of selected virulence genes: $t s s t$ (toxic shock syndrome toxin), sea, seb, sec, seg, seh, sei, sej (staphylococcal enterotoxins A, B, C, G, H, I, J), eta, etb (exfoliative toxins A and B), lukE (LukDE leucocidin), pvl (Panton-Valentine leucocidin, and hla (staphylococcal alpha haemolysin) using PCR with previously published primers [8-10]. The following S. aureus strains were used as positive controls: 2535/07 (eta+, etb+, seg+, sei+), 8977/99 (sea+, sec+, seg+, sei+), 6616/09 (seb+, tsst+, pvl+), 2027/06 (sea+, seh+), and 1034/05 (sea+, seg+, sei+, sej+). The strains used as controls were kindly provided by Prof. Marek Gniadkowski, National Medicines Institute, Warsaw, Poland.

\section{Pulsed-Field Gel Electrophoresis (PFGE)}

Analysis of the genetic similarity between SA isolates was performed by PFGE method in accordance with a previously published protocol [11]. Restriction enzyme digestion was performed with $25 \mathrm{U}$ of SmaI enzyme in Tango buffer (ThermoScientific, USA). Electrophoresis was conducted in a CHEFIII PFGE unit applying the parameters: block 1 - starting pulse $5 \mathrm{~s}$, ending pulse $12 \mathrm{~s}$, voltage $6 \mathrm{~V} / \mathrm{cm}$, run time $11 \mathrm{~h}$, block 2- starting pulse $20 \mathrm{~s}$, ending pulse $60 \mathrm{~s}$, voltage $6 \mathrm{~V} / \mathrm{cm}$, run time $13 \mathrm{~h}$. The GelCompar (Applied Maths, Sint-Martens-Latem, Belgium) was used for cluster analysis using the Dice coefficient and unweighted pair group method with arithmetic mean. Isolates that clustered $\geq 95 \%$ were considered as an epidemic clone. S. aureus strain ATCC 11632 was used as reference.

\section{Spa sequencing}

spa typing was performed as described previously [12], using the spa typing website (http://www.spaserver. ridom.de/) that was developed by Ridom GmbH (Münster, Germany).

\section{SCCmec typing}

Staphylococcal cassette chromosome mec (SCCmec) typing was performed as described previously [13]. The following
S. aureus strains were used as positive controls: ATCCBAA 1762 (SCCmec IV), ATCC-BAA 2094 (SCCmec V), and ATCC-BAA 1681 SCCmec II).

\section{Statistical methods}

The statistical analysis was based on two main groups of techniques. Comparison of the ratio of MSSA/MRSA was performed with a contingency test supplemented by odds ratio analysis. To determine the relationship between continuous and categorical variables, Student's $T$ test and ANOVA were used. If the distribution of the continuous variable did not fit a normal distribution, the analysis was conducted with nonparametric equivalents of parametric tests: Wilcoxon test for Student's $T$ test, and Kruskal-Wallis test for ANOVA. The relationship between two continuous variables was analyzed with Pearson Regression or its non-parametric alternatives. Because of the categorical nature of the effect and combined - numeric as well as categorical types of predictors, the model was constructed for binominal distribution of dependent variables and the logitlinked function. P-values of $<0.05$ were considered to be statistically significant.

\section{Results}

The total number of SA infection was 58 cases, these the incidence of SA-infections was $3.3 \%$ and 1.0/1,000 pds. The incidence of MRSA infections (19 cases) was $1.1 \%$. No significant association was found between time of hospitalization in NICU and the risk of MRSA. The number of MRSA infections in the group of SAinfections - proportion was 32.8 (range from $8.3 \%$ to $42.1 \%$ in different centers). The most common SAinfections were BSI (55.2\%) and pneumonia (39.7\%). MRSA was observed more often among BSI, statistical significance was not observed but a strong trend was noted MRSA ( $\mathrm{p}=0.0515$; odds ratio, OR 2.2807, 95\% Confidence Interval, CI 0.7198-7.2265). MRSA was not associated with a central/peripheral line catheter $(\mathrm{p}=0.8085$, OR $0.963,95 \%$ CI $0.2952-3.1417)$.

All SA-infections were diagnosed on average at day 21 of stay, (median: 17th day). MRSA-infections were diagnosed significantly earlier than MSSA-infections, on average 14th day vs. 23rd day; there was no relationship between the use of devices and prevalence of SA-infections (either MSSA or MRSA).

The fatality case rate was higher among newborns with MRSA infection than with MSSA infection $(10.5 \%$ vs. $0.0 \%)$. The gestational age, birth weight, and other factors of newborns with MRSA was similar to those with MSSA (Table 1).

Multivariate analysis also showed no association between the ward studied, phenotype of the isolate of 
Table 1 Characteristics of newborns with SA-infections

\begin{tabular}{|c|c|c|c|}
\hline & $\begin{array}{l}\text { Infants with MSSA-infections } \\
{[\mathrm{N}=39]}\end{array}$ & $\begin{array}{l}\text { Infants with MRSA-infections } \\
{[\mathrm{N}=19]}\end{array}$ & P-value \\
\hline Risk factors of patients & average $(95 \% \mathrm{Cl})$ & average $(95 \% \mathrm{Cl})$ & \\
\hline Birth weight [grams] & $873.7(791.0-956.5)$ & $948.3(817.3-1079.4)$ & 0.304 \\
\hline Gestational age [weeks] & $27.7(26.8-28.6)$ & $27.2(25.6-28.8)$ & 0.316 \\
\hline CRIB & $5.5(3.5-7.5)$ & $2.4(1.9-6.8)$ & 0.049 \\
\hline Apgar (1 min.) & $4.9(4.3-5.6)$ & $4,7(3.7-5.8)$ & 0.860 \\
\hline Apgar (5 min.) & $6.2(5.6-6.8)$ & $6.0(4.8-7.2)$ & 0.817 \\
\hline length of stay in hospital [days] & $56.2(49.1-63.2)$ & $48.4(37.1-59.8)$ & 0.197 \\
\hline length of hospital stay until infection [days] & $23.8(19.7-28.5)$ & $14.7(9.8-19.7)$ & 0.019 \\
\hline DOT & $11.2(9.6-12.7)$ & $12.3(7.7-16.8)$ & 0.885 \\
\hline DDD & $30.2(22.6-37.8)$ & $26.0(16.4-33.5)$ & 0.457 \\
\hline Risk factors of patients & number (\%) & number (\%) & OR $(95 \% \mathrm{Cl}) \mathrm{P}$-value \\
\hline Female gender & 19 (48.7) & $7(36.8)$ & $0.614(0.200-1.889) 0.393$ \\
\hline Breast feeding & $4(10.5)$ & $1(5.6)$ & $0.486(0.051-4.677) 0.542$ \\
\hline Trophic feeding & $21(55.3)$ & $12(66.7)$ & $1.470(0.477-4.525) 0.418$ \\
\hline Total parenteral nutrition & $25(65.8)$ & $15(79.0)$ & $2.1(0.583-7.571) 0.306$ \\
\hline Fatal cases & $0(0.0)$ & $2(10.5)$ & n/a; n/a; 0.039 \\
\hline
\end{tabular}

CRIB, clinical risk index for babies; DOT, the aggregate sum of the number of days during which at least 1 dose of antibiotic was received; DDD, defined daily dose.

MSSA/MRSA, type of infection, birth weight, and duration of pregnancy.

\section{Antibiotic treatment}

The average antibiotic consumption was similar between MSSA and MRSA cases. The DOT average was 11.2 for MSSA cases and 12.3 for MRSA cases; the DDDs were equal between the groups (30.2 and 26.0, respectively, Table 1). However, statistically significant differences were observed in DOT between the studied wards (ward $B$ was significantly shorter than ward $E, p=0.002$ ) and in DDD (ward F had lower consumption than ward A, $p=0.0049)$. There were no significant differences between DDD and length of therapy of SA-infections depending on phenotype (MRSA vs. MSSA).

Antibiotics used most often for treatment of SA infections (both methicillin-susceptible and -resistant; Table 2) were glycopeptides, beta-lactams and aminoglycosides. For beta-lactams DOT values were significantly higher for MSSA than for MRSA $(Z=-2.77787, p=0.0052$; MRSA mean 3.5; SD 1.37; median 3.5, max 5; for MSSA mean 7.8; SD 4.15; median 7; $\max 21$ ).

Diagram showing antibiotics consumption in the study population is presented in Additional file 1.

Table 2 Distribution of individual antibiotic groups usage in MRSA (18 cases) and MSSA (28 cases) infections

\begin{tabular}{|c|c|c|c|c|c|c|}
\hline \multirow[t]{2}{*}{ Antibiotic groups } & \multicolumn{2}{|c|}{ DOT [\%] } & \multirow[t]{2}{*}{ OR $(95 \% \mathrm{Cl}) \mathrm{P}$-value } & \multicolumn{2}{|c|}{ DDD [\%] } & \multirow[t]{2}{*}{ OR $(95 \% \mathrm{Cl}) \mathrm{P}$-value } \\
\hline & MRSA & MSSA & & MRSA & MSSA & \\
\hline Aminoglycosides & 17.2 & 14.9 & $0.976(0.697-1.367) 0.967$ & 31.7 & 28.0 & $1.366(1.096-1.704) 0.561$ \\
\hline Beta-lactams & 6.3 & 31.7 & $0.146(0.092-0.233) 0.005$ & 19.0 & 40.9 & $0.338(0.264-0.435) 0.879$ \\
\hline Fluoroquinolones & 6.0 & 0.0 & $\mathrm{n} / \mathrm{a}$ & 9.3 & 0.0 & $\mathrm{n} / \mathrm{a}$ \\
\hline Glycopeptides & 63.6 & 43.0 & $2.296(1.766-2.985) 0.427$ & 33.4 & 24.9 & $1.513(1.208-1.895) 0.533$ \\
\hline Lincozamides & 2.7 & 1.1 & $2.562(1.008-6.512) 0.221$ & 0.8 & 1.0 & $0.775(0.250-2.408) 0.073$ \\
\hline Macrolides & 4.2 & 5.8 & $0.723(0.393-1.330) 0.832$ & 2.8 & 4.4 & $0.629(0.348-1.138) 0.072$ \\
\hline Trimethoprim/sulfamethoxazole & 0.0 & 3.0 & $\mathrm{n} / \mathrm{a}$ & 0.0 & 0.2 & $\mathrm{n} / \mathrm{a}$ \\
\hline Metronidazole & 0.0 & 0.5 & $\mathrm{n} / \mathrm{a}$ & 0.0 & 0.6 & $\mathrm{n} / \mathrm{a}$ \\
\hline Total & 100.0 & 100.0 & & 100.0 & 100.0 & \\
\hline
\end{tabular}

OR, odds ratio; $95 \% \mathrm{Cl}, 95 \%$ Confidence Interval; DOT, the aggregate sum of the number of days during which at least 1 dose of antibiotic was received; DDD, defined daily dose; n/a not applicable. 


\section{Resistance and virulence}

The MRSA phenotype was found for 16 isolates (32.6\%) and the inducible MLS (iMLS) phenotype was observed in 11 isolates (22.4\%). Seven strains exhibited both phenotypes. Four isolates (8.2\%) with $\mathrm{M}$ phenotype (resistant to erythromycin but susceptible to clindamycin) were found. All strains were susceptible to ofloxacin, vancomycin and teicoplanin. The $\mathrm{MIC}_{50}$ for vancomycin was $1.5 \mathrm{mg} / \mathrm{ml}$ and for teicoplanin was $1.0 \mathrm{mg} / \mathrm{ml}$.

Molecular characterisation showed that all MRSA isolates carried the mec gene. Only three MLS strains had erm genes (ermA was found in two isolates and ermB in one isolate). One strain had the mup gene.

$S$. aureus isolates were tested for the presence of genes encoding for virulence factors (VFs). The differences in distributions of the VFs were analysed. Detailed data are presented in Table 3: no association was found between number of VFs and resistance to numbers of antimicrobial classes.

Table 3 Virulence factors among isolates and resistance to antimicrobials

\begin{tabular}{|c|c|c|c|}
\hline $\begin{array}{l}\text { Virulence } \\
\text { factor }\end{array}$ & $\begin{array}{l}\text { All }[N=49] \\
(n, \%)\end{array}$ & $\begin{array}{l}\text { MRSA [N = 16]; } \\
(n, \%)\end{array}$ & $\begin{array}{l}\text { MSSA [N = 33]; } \\
(n, \%)\end{array}$ \\
\hline hla & $46(93.9)$ & $16(100)$ & $30(90.9)$ \\
\hline sea & $8(16.3)$ & $1(6.3)$ & 7 (21.2) \\
\hline seb & 0 & 0 & 0 \\
\hline sec & 7 (14.3) & $4(25)$ & $3(9.1)$ \\
\hline seg & $32(65.3)$ & $15(93.8)$ & $17(51.5)$ \\
\hline seh & $1(2.0)$ & $1(6.3)$ & 0 \\
\hline sei & $32(65.3)$ & $15(93.8)$ & $17(51.5)$ \\
\hline sej & $4(8.2)$ & 0 & $4(12.1)$ \\
\hline eta & 7 (14.3) & $4(25)$ & $3(9.1)$ \\
\hline etb & 0 & 0 & 0 \\
\hline tsst & $9(18.4)$ & 0 & $9(27.3)$ \\
\hline lukE & 19 (38.8) & 0 & 19 (57.6) \\
\hline pvl & 0 & 0 & 0 \\
\hline Resistance & All [N = 49]; (\%) & MRSA [N = 16]; (\%) & MSSA [N = 33]; (\%) \\
\hline E & $16(32.6)$ & $10(62.5)$ & $6(18.2)$ \\
\hline DA & $12(24.5)$ & $7(43.7)$ & $5(15.1)$ \\
\hline OFX & 0 & 0 & 0 \\
\hline GN & $3(6.1)$ & $2(12.5)$ & $1(3.0)$ \\
\hline TOB & $6(12.2)$ & $2(12.5)$ & $4(12.1)$ \\
\hline AK & $6(12.2)$ & $3(18.7)$ & $3(9.1)$ \\
\hline VA & 0 & 0 & 0 \\
\hline TEC & 0 & 0 & 0 \\
\hline
\end{tabular}

Legend: E-erythromycin, DA-clindamycin, OFX - ofloxacin, GN - gentamycin, TOB - tobramycin, AK - amikacin, VA - vancomycin, TEC - teicoplanin. hla (staphylococcal alpha haemolysin), sea, seb, sec, seg, seh, sei, sej (staphylococcal enterotoxins $A, B, C, G, H, I, J)$, eta, etb (exfoliative toxins $A$ and $B$ ), tsst (toxic shock syndrome toxin), lukE (LukDE leucocidin), pvl (Panton-Valentine leucocidin).

\section{Typing}

MSSA isolates showed very different pulsotypes; dominant clones were not detected. Cluster analysis based on PFGE of the 33 isolates showed 23 pulsotypes, some of which were less than $70 \%$ similar, suggesting a genotypically variable population. Isolates with identical pulsotypes were usually derived from different patients in the same NICU during the same period of time. Analysis of SmaI macro-restriction profiles of the 16 MRSA isolates revealed one dominant clone (14 isolates). The two remaining MRSA isolates had different PFGE patterns (Figure 1). MRSA isolates belonging to the dominant clone were derived from one NICU designed by the letter F. Two spa types and two SCCmec were observed: spa-t015 in the case of 15 isolates belonging to the SCCmec type IV and spa-t011 in one isolate belonging to the SCCmec type V.

\section{Discussion}

Staphylococcus aureus is still one of the most important causes of infections in NICU patients, and usually represents a large part of the aetiology of infection. For example, in the UK, Vergnano reported a prevalence of about $18 \%$ [14], which was similar to the level in the U.S. [15], whereas in Germany the prevalence was $10.2 \%$ [16]. In the group of neonates in the present study, SA-infections were observed 2-3 times less frequently. Similar results have been obtained in the field of drug resistance, especially MRSA, in late- and early-onset infections. A number of multicentre studies, however, found a higher proportion of MRSA. In Japan, MRSA strains accounted for more than $88 \%$ [17] of SA infections, and in Cyprus, 68\% [18], of SA infections.

In contrast, in the US (only in VLBW newborns) the prevalence of MRSA infection was $0.4 \%$ [19], while in Poland and the UK, it was $1.1 \%$ [14]. Furthermore, according to Vergnano's data, the proportion of infections with the MRSA phenotype in late infections was a little over $10 \%$ of SA-infections, which was much less than in the group examined in the present study [14].

The difference in findings between studies may be due to the fact that the United Kingdom was one of the first countries in the world to introduce wide mandatory surveillance of MRSA [20,21]. For example, beginning in 2010, screening for MRSA in most patients admitted for elective hospital was instituted [22]; this action underscores the fact that MRSA colonization was considered an important risk factor for this aetiology of infection [23]. Implementation of the UK guidelines resulted in a reduction of MRSA infections in health care settings in general [24]. Furthermore, this reduction has had a direct impact on the prevalence of MRSA in the general population; thus, the risk of colonization of pregnant women, young parents and NICU staff also has dropped 


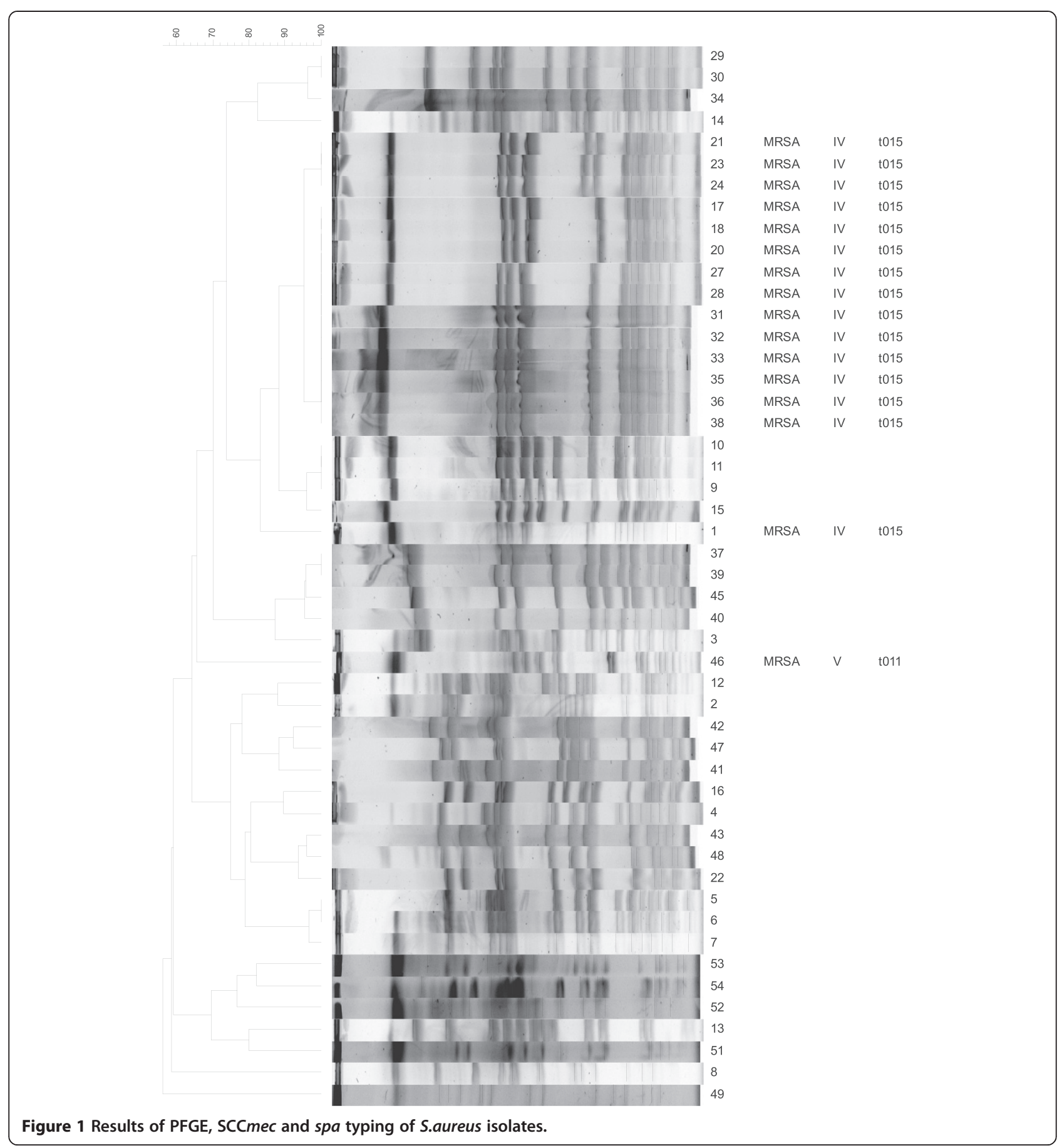

especially since MRSA is not only found in hospitals, but often occurs as "community-acquired" infections [25,26].

The fact that there was no relationship between MRSA infections resulting from invasive procedures and lateonset MRSA infections should be discussed with special attention. A number of authors pointed to an increasing risk of infections with multidrug-resistant (MDR) strains during hospitalisation and resulting from invasive procedures. Lessa et al. showed that MRSA infections among newborns observed most often were late-onset: median age at disease onset was 28 days [19]. These results suggest that infection with MDR strains should be also considered at the early stage of hospitalisation. No relationship between MRSA infections resulting from invasive procedures could be made with the huge number of invasive procedures among this group of patients. Hocevar et al. showed that MRSA constituted about $33 \%$ of device-associated infections, which is consistent with the results of this study [27]. 
Rational antimicrobial treatment is an important element of surveillance for drug-resistant strains [28]. The results of this study reveal the methodological difficulties associated with the assessment of the consumption of antibiotics by neonates. In similar studies, other authors used indicators such as DOT, LOT (the number of days where at least one dose of any antibiotic was received), DDD or PDD (prescribed daily dose) [29,30]. In the present study, there were no significant differences in DOT or DDD as determined by analysis of the average consumption of all groups of antibiotics used in the treatment of SA infections. Stratification of antibiotic consumption according to individual groups showed, however, that the indicator used, (DOT/DDD), has an impact on significance of the differences in therapeutic regimens used for MRSA and MSSA. In case of beta-lactams the level of antibiotic consumption was statistically significantly higher only in case of assessment with DOT usage. Therefore, analysis of antibiotic consumption should not be based on only one parameter, and the data should be stratified in order to be able to determine meaningful differences [31].

The data about antibiotic consumption show high usage of glycopeptides in both groups: MRSA-infections and MSSA-infections, when expressed as DOT. Rapid diagnostic molecular methods (which enable rapid assessment of the need to implement or discontinue therapy with vancomycin) should be implemented to decrease glycopeptide consumption, because glycopeptides are not easy to use in neonates [32,33]. On the other hand, the study of Brzychczy-Włoch et al. showed a high percentage of Gram-positive mecA-positive infections in NICUs [34]. For this reason vancomycin should not be eliminated from use among NICUs but effort should be made toward lowering the vancomycin consumption.

As expected, the haemolysin gene was present in almost all studied isolates with occasional exceptions. In contrast, genes encoding exfoliative toxins were rare: no such patients were among our study population. The low frequency of eta and etb coincides with the result of the other studies [2,35].

In recent years, MRSA producing the Panton-Valentine leucocidin toxin have emerged as increasingly common causes of community-acquired infections [36,37]; however, we did not find $p v l$-positive isolates, which was similar to other studies [24].

Of enterotoxin genes detected, those within the egc (enterotoxin gene cluster) seg $+s e i$ were the most frequent $(65.3 \%)$, which also was shown by Rasmussen [35]. These enterotoxin genes were found frequently in isolates from patients but their presence did not mean the production of toxin was high enough to cause food poisoning. These superantigens may enhance the persistence of infection but their role as a virulence factors is still purely speculative [38].
Among leucocidins, the prevalence of $l u k E$ was relatively high and associated with MSSA but not MRSA strains. Moreover, the tsst gene was not detected in any of the MRSA isolates, which was similar to other studies [2].

There have been some studies showing a link between virulence and resistance $[39,40]$, no such association was found in this study.

A number of molecular methods have been developed and implemented for typing MRSA isolates including SCCmec, PFGE, and spa typing [2]. Most of the MRSA strains (and only MRSA) isolated in this study belonged to the epidemic clone: these strains had identical pulsotypes, belonged to the same SCCmec type and spa type, and came from one of the surveyed centers.

Reports from NICUs showed that it is not easy to determine the incidence of MRSA-infections. However, results from the present study, combining detailed epidemiological analyses with molecular studies of SA, allowed a much broader evaluation of these infections: development, course and severity of the infection were compared with resistance and virulence of the strain, and the type of populations where they were observed.

Several potential limitations should be considered in the interpretation of data presented here. First, the number of isolates investigated was low. Another important limitation was the lack of a control group - surveillance was conducted only on a group of very-low birth weight infants.

\section{Conclusions}

The key points of results of our study are:

- the MRSA infections were diagnosed significantly earlier than MSSA-infections (14 vs. 23 day of hospitalization)

- low proportion of MRSA infections in the group of SA-infections (32.8\%) which additionally were less frequent than reported in other studies pointing at specific epidemiological situation in Polish NICU (more detailed studies are recommended)

- high usage of glycopeptides in the MSSA infections treatment which indicates the necessity of antimicrobial stewardship improvement and introducing molecular screening for early identification of infections

\section{Additional file}

Additional file 1: Antibiotics consumption in the study population.

Competing interests

The authors declare that they have no competing interests. 


\section{Authors' contributions}

JWM designed the study, analysed and interpreted the epidemiological data, drafted the manuscript and financially supported the study, AR analysed the antibiotic consumption data and drafted the manuscript, DR carried out the antimicrobial susceptibility studies, ACH carried out the molecular genetic studies, drafted the manuscript, MP drafted the manuscript, PA performed the statistical analysis, RL, MBK, EG, EH and AK collected and participated in study design, MB have given final approval of the version to be published. All authors read and approved the final manuscript.

\section{Acknowledgements}

Financial support. The research project was conducted under the supervision of Jadwiga Wojkowska-Mach. This work was supported by a grant from the Ministry of Science and Higher Education. - the grant number: DEC-2011/03/B/NZ7/01911. The sponsor provided the funding for the project only.

\section{Author details}

${ }^{1}$ Chair of Microbiology, Jagiellonian University Medical College, 18 Czysta Street, Kracow 31-121, Poland. Institute of Nature Conservation, Polish Academy of Sciences, Kraków, Poland. ${ }^{3}$ Clinic of Neonatology and Intensive Neonatal Care, Institute of Mother and Child, Warsaw, Poland. ${ }^{4}$ Clinic of Neonatology, Jagiellonian University Medical College, Kraków, Poland. ${ }^{5}$ Clinic of Neonatology and Intensive Neonatal Care, Warsaw Medical University, Warsaw, Poland. ${ }^{6}$ Clinic of Neonatology, Polish Mother's Memorial Hospital-Research Institute, Lodz, Poland. 'Department of Neonatal Diseases, Pomeranian Medical University, Szczecin, Poland.

\section{Received: 25 November 2014 Accepted: 12 March 2015} Published online: 01 April 2015

\section{References}

1. Stoll BJ, Hansen N, Fanaroff AA, Wright LL, Carlo WA, Ehrenkranz RA, et al. Late-onset sepsis in very low birth weight neonates: the experience of the NICHD Neonatal Research Network. Pediatrics. 2002;110:285-91.

2. Machuca MA, Sosa LM, González Cl. Molecular typing and virulence characteristic of methicillin-resistant Staphylococcus aureus isolates from pediatric patients in Bucaramanga, Colombia. PLoS One. 2013;8(8):e73434.

3. Gastmeier P, Geffers C, Schwab F, Fitzner J, Obladen M, Rüden H. Development of a surveillance system for nosocomial infections: the component for neonatal intensive care in Germany. J Hosp Infect. 2004;57:126-33.

4. Rich $M$, Deighton $L$, Roberts $L$. Clindamycin-resistance in methicillin-resistant Staphylococcus aureus isolated from animals. Vet Microbiol. 2005;111:237-40.

5. Pereira EM, Schuenck RP, Malvar KL, lorio NL, Matos PD, Olendzki AN, et al. Staphylococcus aureus, Staphylococcus epidermidis and Staphylococcus haemolyticus: methicillin-resistant isolates are detected directly in blood cultures by multiplex PCR. Microbiol Res. 2010;165:243-9.

6. Sutcliffe J, Grebe T, Tait-Kamradt A, Wondrack L. Detection of erythromycinresistant determinants by PCR. Antimicrob Agents Chemother. 1996;40 (11):2562-6.

7. Anthony RM, Connor AM, Power EG, French GL. Use of the polymerase chain reaction for rapid detection of high-level mupirocin resistance in staphylococci. Eur J Clin Microbiol Infect Dis. 1999;18:30-4.

8. Johnson WM, Tyler SD, Ewan EP, Ashton FE, Pollard DR, Rozee KR. Detection of genes for enterotoxins, exfoliative toxins, and toxic shock syndrome toxin 1 in Staphylococcus aureus by the polymerase chain reaction. J Clin Microbiol. 1991;29(3):426-30.

9. Løvseth A, Loncarevic S, Berdal KG. Modified multiplex PCR method for detection of pyrogenic exotoxin genes in staphylococcal isolates. J Clin Microbiol. 2004;42(8):3869-72.

10. Lina G, Piémont Y, Godail-Gamot F, Bes M, Peter MO, Gauduchon V, et al. Involvement of Panton-Valentine leukocidin-producing Staphylococcus aureus in primary skin infections and pneumonia. Clin Infect Dis. 1999;29(5):1 128-32.

11. McDougal LK, Steward CD, Killgore GE, Chaitram JM, MCAllister SK, Tenover FC. Pulsed-field gel electrophoresis typing of oxacillin-resistant Staphylococcus aureus isolates from the United States: establishing a national database. J Clin Microbiol. 2003;41:5113-20.

12. Harmsen D, Claus H, Witte W. Typing of methicillin-resistant Staphylococcus aureus in a university hospital setting by using novel software for spa repeat determination and database management. J Clin Microbiol. 2003;41(12):5442-8

13. Kondo $Y$, Ito T, Ma XX, Watanabe S, Kreiswirth BN, Etienne J, et al. Combination of multiplex PCRs for staphylococcal cassette chromosome mec type assignment: rapid identification system for mec, ccr, and major differences in junkyard regions. Antimicrob Agents Chemother. 2007;51(1):264-74.

14. Vergnano S, Menson E, Kennea N, Embleton N, Russell AB, Watts T, et al. Neonatal infections in England: the NeonIT surveillance network. Arch Dis Child Fetal Neonatal Ed. 2011;96:F9-14.

15. Schrag S, Gorwitz R, Fultz-Butts K, Schuchat A. Prevention of perinatal group $B$ streptococcal disease. Revised guidelines from CDC. Morb Mortal Wkly Rep. 2002;15:1-22

16. Geffers C, Baerwolff S, Schwab F, Gastmeier P. Incidence of healthcareassociated infections in high-risk neonates: results from the Germen surveillance system for very-low-birth weight infants. J Hosp Infect. 2008;68(3):214-21.

17. Babazono A, Kitajima H, Nishimaki S, Nakamura T, Shiga S, Hayakawa M, et al. Risk factors for nosocomial infections in the neonatal intensive care unit by the Japanese National Infection Surveillance (JANIS). Acta Med Okayama. 2008;62(4):261-8

18. Gikas A, Roumbelaki M, Bagatzouni-Pieridou D, Alexandrou M, Zinieri V, Dimitriadis I, et al. Device-associated infections in the intensive care units of Cyprus: results of the first national incidence study. Infection. 2010:38:165-71.

19. Lessa FC, Edwards JR, Fridkin SK, Tenover FC, Horan TC, Gorwitz RJ. Trends in incidence of late-onset methicillin-resistant Staphylococcus aureus infection in neonatal intensive care units: data from the National Nosocomial Infections Surveillance System, 1995-2004. Pediatr Infect Dis J. 2009;28(7):577-81.

20. Health Protection Agency: Mandatory Surveillance of Healthcare Associated Infections Report 2006. http://www.hpa.org.uk/web/ HPAwebFile/HPAweb_C/1194947323575; Accessed: 27.05.2011.

21. Coia E, Duckworth GJ, Edwards DI, Farrington M, Fry C, Humphreys H, et al. Guidelines for the control and prevention of methicillin-resistant Staphylococcus aureus (MRSA) in healthcare facilities. J Hosp Infect. 2006;66(S1):1-44.

22. Hawkins G, Stewart S, Blatchford O, Reilly J. Should healthcare workers be screened routinely for methicillin-resistant Staphylococcus aureus? A review of the evidence. J Hosp Infect. 2011;77:285-9.

23. Maraqa NF, Aigbivbalu L, Manisa-lusan C, Wludyka P, Shareef Z, Bailey C, et al. Prevalence of and risk factors for methicillin-resistant Staphylococcus aureus colonization and infection among infants at a level III neonatal intensive care unit. Am J Infect Control. 2011;39:35-41.

24. European Antimicrobial Resistance Surveillance System: EARSS Annual Report 2008. http://ecdc.europa.eu/en/activities/surveillance/EARS-Net/ Documents/2008_EARSS_Annual_Report.pdf (Accessed 27.05.2011).

25. Kuint J, Barzilai A, Regev-Yochay G. Comparison of community-acquired methicillin-resistant Staphylococcus aureus bacteremia to other staphyloccocal species in a neonatal intensive care unit. Eur J Pediatr. 2007;166:319-25.

26. CDC. Community-associated methicillin resistant Staphylococcus aureus infection among healthy newborns - Chicago and Los Angeles Country, 2004. MMWR Morb Mortal Wkly Rep. 2006;55(12):329-32.

27. Hocevar SN, Edwards JR, Horan TC, Morrell GC, Iwamoto M, Lessa FC. Device-associated infections among neonatal intensive care unit patients: incidence and associated pathogens reported to the National Healthcare Safety Network, 2006-2008. Infect Control Hosp Epidemiol. 2012;33(12):1200-6.

28. Livermore DM. Bacterial resistance: origins, epidemiology and impact. CID. 2003:36:11-23

29. Porta A, Hsia Y, Doerhoit K, Spyridis N, Bielicki J, Menson E, et al. Comparing neonatal and paediatric antibiotic prescribing between hospitals: a new algorithm to help intenational benchmarking. J Antimicrob Chemother. 2012;67:1278-86

30. Hersh AL, Newland JG, Metjian TA, Gerber JS, Kronman MP, Ross RK, et al. Identifying targets for antimicrobial stewardship in children's hospital. Infect Control Hosp Epidemiol. 2013;34(12):1252-8.

31. Berrington A. Antimicrobial prescribing in hospitals: be careful what you measure. J Antimicrob Chemother. 2010;65:163-8.

32. Lim HS, Chong YP, Noh YH, Jung JA, Kim YS. Exploration of optimal dosing regimens of vancomycin in patients infected with methicillin-resistant Staphylococcus aureus by modeling and simulation. J Clin Pharm Ther. 2014;16.39(2):196-203.

33. Ampe E, Delaere B, Hecq JD, Tulkens PM, Glupczynski Y. Implementation of a protocol for administration of vancomycin by 
continuous infusion: pharmacokinetic, pharmacodynamic and toxicological aspects. Int J Antimicrob Agents. 2013;41(5):439-46.

34. Brzychczy-Wloch M, Borszewska-Kornacka M, Gulczynska E, Wojkowska-Mach J, Sulik M, Grzebyk M, et al. Prevalence of antibiotic resistance in multi-drug resistant coagulase-negative staphylococci isolated from invasive infection in very low birth weight neonates in two polish NICUs. Ann Clin Microbiol Antimicrobial. 2013;12:41.

35. Rasmussen G, Monecke S, Ehricht R, Söderquist B. Prevalence of clonal complexes and virulence genes among commensal and invasive Staphylococcus aureus isolates in Sweden. PLoS One. 2013;8(10):e77477.

36. Ali H, Nash JQ, Kearns AM, Pichon B, Vasu V, Nixon Z, et al. Outbreak of a South West Pacific clone Panton-Valentine leucocidin-positive methicillin-resistant Staphylococcus aureus infection in a UK neonatal intensive care unit. J Hosp Infect. 2012;80(4):293-8.

37. McAdams RM, Mazuchowski E, Ellis MW, Rajnik M. Necrotizing staphylococcal pneumonia in a neonate. J Perinatol. 2005;25(10):677-9.

38. Adwan G, Adwan K, Jarrar N, Salama Y, Barakat A. Prevalence of seg, she and sei genes among clinical and nasal Staphylococcus aureus isolates in Palestine. Br Microbiol Res J. 2013;3(2):139-49.

39. Jiang W, Zhou Z, Zhang K, Yu Y. Epidemiological investigation of community-acquired Staphylococcus aureus infection. Genet Mol Res. 2013;12(4):6923-30.

40. Lewandowski T, Huang J, Fan F, Rogers S, Gentry D, Holland R, et al. Staphylococcus aureus formyl-methionyl transferase mutants demonstrate reduced virulence factor production and pathogenicity. Antimicrob Agents Chemother. 2013;57(7):2929-36.

\section{Submit your next manuscript to BioMed Central and take full advantage of:}

- Convenient online submission

- Thorough peer review

- No space constraints or color figure charges

- Immediate publication on acceptance

- Inclusion in PubMed, CAS, Scopus and Google Scholar

- Research which is freely available for redistribution 